\title{
Influence des couvertures lithologiques et végétales sur les régimes et la qualité des eaux des affluents congolais du fleuve Congo
}

\section{Impact of lithological and vegetation covers on flow discharge and water quality of Congolese tributaries of the Congo river}

\author{
A. Laraque, M. Mietton, J. C. Olivry et A. Pandic
}

Volume 11, numéro 2, 1998

URI : https://id.erudit.org/iderudit/705304ar

DOI : https://doi.org/10.7202/705304ar

\section{Aller au sommaire du numéro}

\section{Éditeur(s)}

Université du Québec - INRS-Eau, Terre et Environnement (INRS-ETE)

ISSN

0992-7158 (imprimé)

1718-8598 (numérique)

\section{Découvrir la revue}

Citer cet article

Laraque, A., Mietton, M., Olivry, J. C. \& Pandic, A. (1998). Influence des couvertures lithologiques et végétales sur les régimes et la qualité des eaux des affluents congolais du fleuve Congo. Revue des sciences de l'eau / Journal of Water Science, 11(2), 209-224. https://doi.org/10.7202/705304ar

\section{Résumé de l'article}

Les affluents et sous affluents congolais du fleuve Congo se partagent en deux systèmes hydrologiques, qui, tout en étant proches dans l'espace, sont sensiblement différents dans leur fonctionnement. Cette distinction repose sur la différenciation manifeste entre les deux régions naturelles que sont les "Plateaux Tékés" d'une part et la "Cuvette congolaise" d'autre part, nettement contrastées du point de vue du couvert végétal et lithologique et subsidiairement du climat

La classification hydrologique que nous présentons est basée sur l'étude des coefficients d'écoulement interannuel (rapport de la lame d'eau écoulée sur la lame d'eau précipitée, exprimé en pourcentage) et sur le coefficient d'irrégularité saisonnière moyen des débits qui est le rapport moyen sur les chroniques disponibles, entre débits mensuels maximum et débits mensuels minimum par année hydrologique. Pour les rivières Tékés, le premier paramètre présente des valeurs comprises entre $45 \%$ et $60 \%$ contre $20 \%$ à $30 \%$ pour la "Cuvette Congolaise", alors que le deuxième paramètre varie respectivement pour ces deux régions de 1,1 à 1,5 et de 2,5 à 5,5.

Cette classification présente l'avantage d'être à la fois adaptée à ces deux contextes régionaux voisins mais aussi de mettre en évidence le rôle prépondérant des formations géologiques et des couvertures végétales sur les écoulements des cours d'eau concernés, ainsi que sur la qualité des eaux.

En effet, après son passage au travers d'un important aquifère gréseux, les eaux des Plateaux Tékés à couverture de savane font partie des "eaux claires", à très faible minéralisation, marquée essentiellement par la présence de silice dissoute. En revanche, les " eaux noires " couleur de thé qui drainent la Cuvette Congolaise, couverte de forêts, sont très riches en matière organique et très acides 


\title{
Influence des couvertures lithologiques et végétales sur les régimes et la qualité des eaux des affluents congolais du fleuve Congo
}

\author{
Impact of lithological and vegetal covers on flow \\ discharge and water quality of Congolese tributaries \\ from the Congo river
}

\author{
A. LARAQUE ${ }^{*}$, M. MIETTON 2 , J.C. OLIVRY ${ }^{3}$ et A. PANDI ${ }^{4}$
}

Reçu le 27 août 1996, accepté le 23 décembre 1997**,

\section{SUMMARY}

The Congolese tributaries and sub-tributaries of the Congo river come from two adjacent regions that have similar annual rainfall volumes $(1,700$ and $1,900 \mathrm{~mm} \mathrm{year}^{-1}$ ) but very different physiographic characteristics. These latter characteristics are the main reasons accounting for the differences in their hydrological behavior. The purpose of this work is to introduce a regional hydrological classification - adapted to the particularities of the regions that are crossed by these tributaries - that points out the major influence of the geological formations and the vegetative cover on the runoff of the rivers, as well as on their water qualities. The two natural regions concerned are the Téké plateaus and the Congolese basin. The first region, centered on Congo but also spread into Gabon in its western part and into Congo on its eastern side, consists of sand-gritty formations of the Tertiary going from 200 to $400 \mathrm{~m}$ in thickness, covered with savanna. The second region builds up a continuation of the wide Congo depression that occupies the whole center of the Congo river basin. It comprises Quaternary alluvial deposits, covered with swamps and dense equatorial rain forests. The hydrological characterization we present here is based on two hydrological coefficients that do not have physical linkages but illustrate the differences in the regimes of their respective flows. These are the interannual runoff coefficient (KE) and the average seasonal variability of the discharges (CIS) observed over a period of forty years. Concerning the Téké rivers, the first parameter (KE) has values ranging between $45 \%$ and $60 \%$ versus $20 \%$ o $30 \%$ for the Congolese basin. The second parameter (CIS) has values ranging from 1.1 to 1.5 for the Téké rivers, versus 2.5 to 5.5 for the Congolese basin. The specific discharges of the first region vary from 25 to $35 \mathrm{~L} . \mathrm{s}^{-1} \cdot \mathrm{km}^{-2}$ versus 10 and $15 \mathrm{~L} \cdot \mathrm{s}^{-1} \cdot \mathrm{km}^{-2}$ for the second region. Concerning

1. ORSTOM, CP 7091, Lago Sul, CEP 71619-970 Brasilia, DF - Brasil.

2. CEREG, Université L. PASTEUR, 3 rue de l'Argonne, 67083 Strasbourg, France.

3. ORSTOM, BP 5045, 34032 Montpellier cedex 1 , France.

4. ORSTOM, BP 181, Brazzaville, Congo.

* Correspondance. e-mail : laraque@dnaee.gov.br.

** Les commentaires seront reçus jusqu’au 31 décembre 1998. 
the Téké plateaus, the weak average seasonal variations of the discharges can only be explained by a great capacity for storage, and consequently for flow regulation, of the sand-gritty aquifer. Infiltration of the rainfall waters is very important in this thickness aquifer which attenuates the floods and helps minimize the droughts this important aquifer which attenuates the floods and helps minimize the droughts. This is also the reason why it is in this region that some of the most regular rivers of the planet are encountered. Concerning the Congolese basin, the lower permeability of the soils, the interception of rain waters, the evapotranspiration of the forest cover, and the direct evaporation on the floodable areas as well as the swamps, lead to an important water deficit. As there is no aquifer, at least none as important and regulating as in the Téké plateaus, the hydrological regime is more similar to the regional rainfall rhythm. The Téké hydrological regimes are for their part practically independent of the regional rainfall regime. From a qualitative point of view, these waters are also very distinet. Indeed, after crossing the important gritty aquifer, the waters of the Téké plateau - which is covered with savanna - consist of highly diluted clear waters, relatively rich in dissolved silica. These waters, with dissolved inorganic matter ranging from 1 to $3 \mathrm{mg} \cdot \mathrm{L}^{-1}$ (not taking into account the dissolved silica), are similar in composition to rainfall waters. These are among the most dilute surface waters of the world, the soluble ions of the mentioned formations having been almost totally leached. On the other hand, waters draining the Congolese basin originate from a long residence time under the forest cover. They are very rich in organic matter (up to $44 \%$ of particulate organic carbon) and very acid (pH can be lower than 4). They are truly qualified as "black rivers". Their mineralization, although low (11 to $30 \mathrm{mg} . \mathrm{L}^{-1}$, without dissolved silica), can be considered high when compared to the plateaus' waters, and also of a greater diversity. For both regions, the content in dissolved silica is identical to the world wide average, varying between 8 and $10 \mathrm{mg.L} \mathrm{L}^{-1}$. If the specific export rates in dissolved matter are low, they are nevertheless identical in the two geographical domains despite the important differences in concentrations. For matter of mineral and organic origins, we calculate respectively $10 \mathrm{t.km}-2$.year-1 in the basin versus $9.7 \mathrm{t} . \mathrm{km}^{-2}$.year-1 on

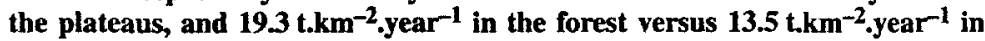
the savanna. This apparent similitude is obviously linked to the compensation by the specific discharges of the Plateau rivers, more than twice as much as in the basin.

Key-words: hydrological regime, hydrograph, runoff coefficient, specific discharge, module, congo, téké plateaus, congolese basin, water qualing:

Les affuents et sous affiuents congolais du fleuve Congo se partagent en deux systèmes hydrologiques, qui, tout en étant proches dans l'espace, sont sensiblement différents dans leur fonctionnement. Cette distinction repose sur la différenciation manifeste entre les deux régions naturelles que sont les « Plateaux Tékés * d'une part et la * Cuvette congolaise * d'autre part, nettement contrastées du point de vue du couvert végétal et lithologique et subsidiairement du climat. La classification hydrologique que nous présentons est basée sur l'étude des coefficients d'écoulement interannuel (rapport de la lame d'eau écoulée sur la lame d'eau précipitée, exprimé en pourcentage) et sur le coefficient d'irrégularité saisonnière moyen des débits qui est le rapport moyen sur les chroniques disponibles, entre débits mensuels maximum et débits mensuels minimum par année hydrologique. Pour les rivières Tékés, le premier paramètre présente des valeurs comprises entre $45 \%$ et $60 \%$ contre $20 \%$ à $30 \%$ pour la « Cuvette Congolaise *, alors que le deuxième paramètre varie respectívement pour ces deux régions de 1,1 à 1,5 et de 2,5 à 5,5. Cette classification présente l'avantage d'être à la fois adaptée à ces deux contextes régionaux voi- 
sins mais aussi de mettre en évidence le rôle prépondérant des formations géologiques et des couvertures végétales sur les écoulements des cours d'eau concernés, ainsi que sur la qualité des eaux. En effet, après son passage au travers d'un important aquifêre gréseux, les eaux des Plateaux Tékés à couverture de savane font partie des " eaux claires ", à très faible minéralisation, marquée essentiellement par la présence de silice dissoute. En revanche, les * eaux noires $»$ couleur de thé qui drainent la Cuvette Congolaise, couverte de forêts, sont très riches en matière organique et très acides.

Mots clés : régime hydrologique, hydrogramme, coefficient d'écoulement, débit spécifique, module, congo, plateaux tékés, cuvette congolaise, qualité des eaux.

\section{INTRODUCTION}

Les affluents congolais de rive droite du Congo drainent toute la partie occidentale et septentrionale de son bassin versant. Leurs confluences se situent le long d'un tronçon de $600 \mathrm{~km}$, de direction globale nord-sud, en amont de Brazzaville (figure 1). Ils proviennent de deux régions bien individualisées dont nous présentons les caractéristiques physiographiques avant d'aborder les particularités des régimes hydrologiques qu'elles induisent. Dans le cadre d'une synthèse des résultats existants, nous tentons ensuite d'établir une classification hydrologique régionale permettant d'expliquer et de commenter leurs dynamiques de fonctionnement hydrologique ainsi que la qualité de leurs eaux d'écoulement.

\section{1 - RÉGION ET DONNÉES}

\subsection{Région étudiée}

La région dite des « Plateaux Tékés ou Batékés " s'étend au nord de Brazzaville (figure 1) sous un climat équatorial (figure 2a), dont le total pluviométrique annuel moyen est d'environ $1900 \mathrm{~mm}$. Ces plateaux proprement dits ou collines très arrondies, de 700 à $800 \mathrm{~m}$ d'altitude environ, sont caractérisés par des écoulements superficiels inexistants. Cette particularité s'explique par la nature très perméable et la forte épaisseur, de 200 à 400 mètres, des formations sablo-gréseuses du Kalahari qui les composent. D'âge tertiaire, elles comprennent les deux niveaux Ba1 $(300 \mathrm{~m})$ et Ba2 (20 à $90 \mathrm{~m})$ qui correspondent respectivement aux grès polymorphes (Paléogène) et aux sables ocres (Néogène) du Zaïre (CoSSON, 1955 ; SCHWARTZ, 1988). Ils constituent au Congo, un puissant aquifère de $45000 \mathrm{~km}^{2}$, principal château d'eau du pays, qui alimente des vallées profondes le long desquelles serpentent des forêts galeries. Ces séries se prolongent à l'ouest comme à l'est, en territoires gabonais et zaïrois, pour courrir une superficie d'environ $110000 \mathrm{~km}^{2}$.

Malgré la pluviosité élevée, les brûlis pratiqués par l'homme ajoutent leurs effets à la perméabilité du substratum et à la complète infiltration des eaux météoriques pour limiter la végétation à une simple savane herbeuse à Ludétia demeusii, piquetée d'arbustes. 


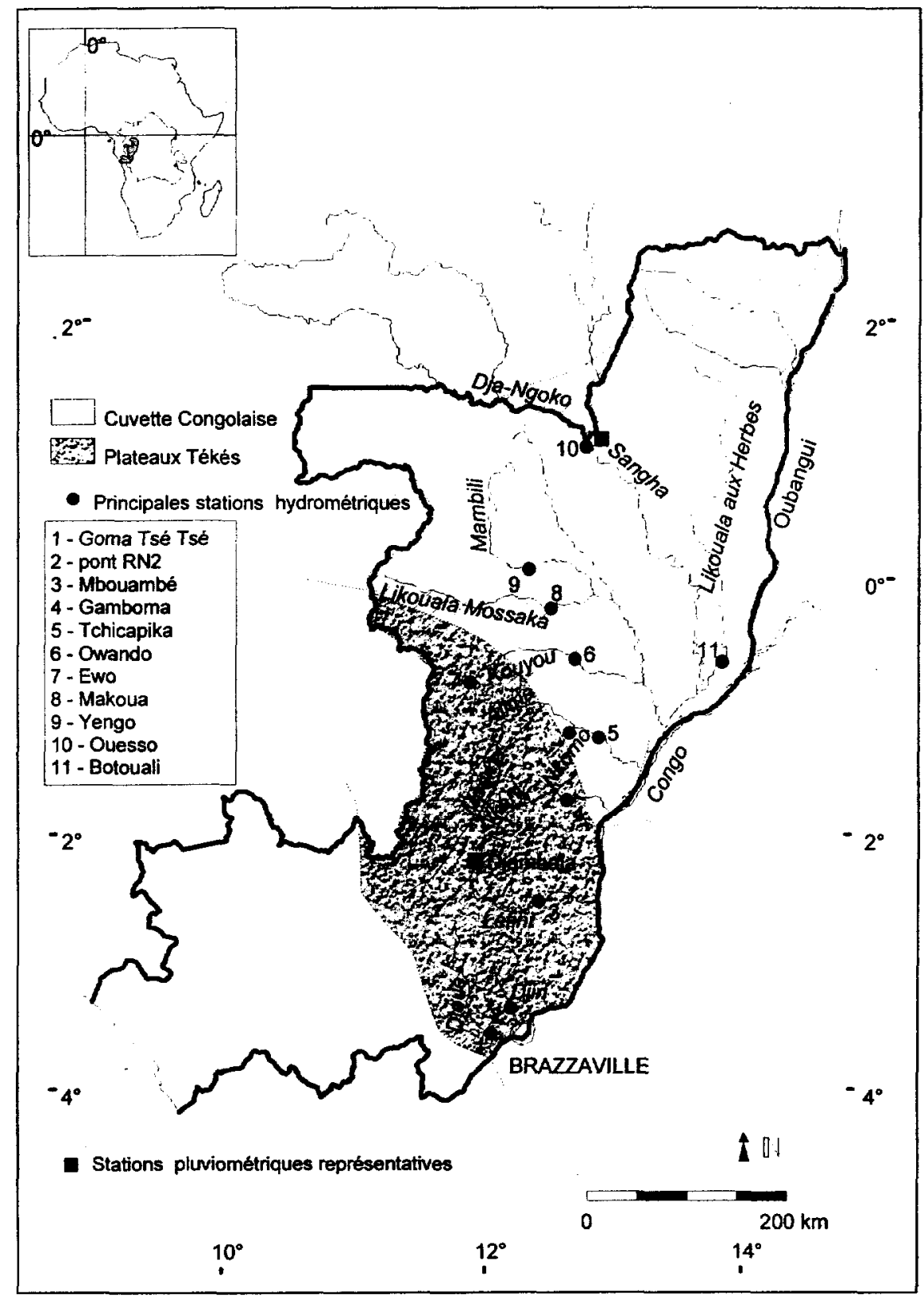

Figure 1 Affluents congolais du fleuve Congo et contours (lissés) des plateaux "Tékés" et de la " Cuvette".

Congolese tributaries of the Congo river and smoothed contours of the Tekes plateaus and of the Congolese basin. 
Au Nord - Nord Est du territoire Téké et jusqu'à la frontière avec la République Centrafricaine, s'étend la région dénommée "Cuvette Congolaise " dont le point le plus bas à environ 400 mètres d'altitude est constitué par l'important " carrefour " de confluences avec le Congo, de l'Oubangui, la Sangha, la Likouala aux Herbes et la Likouala Mossaka. Elle est le prolongement de la vaste dépression Congolaise dont la seule partie inondable à $60 \%$ de sa superficie, couvre près de $220000 \mathrm{~km}^{2}$ selon BURGIS et SYMOENS (1987). Elle correspond à un domaine pouvant être facilement distingué du précédent, puisque domine là une forêt ombrophile, dense et humide, en partie inondée en période de crue, aux côtés de prairies et de marécages. Les cours d'eau de cette plaine inondable sont très sinueux, parfois anastomosés à proximité de leur confluence et reliés entre eux par de nombreux chenaux naturels ou anthropiques. Les limites de partage des eaux entre bassins versants sont parfois très délicates à établir en raison de la faiblesse du relief et les écoulements dans ces canaux peuvent d'ailleurs localement s'inverser au gré des fluctuations du niveau des rivières qu'ils desservent. Au-delà des bourrelets de berge, les eaux d'épandage se mélangent souvent avec celles du toit de la nappe alluviale qui affleure à maintes reprises, l'ensemble s'écoulant lentement vers l'aval de manière laminaire. POUYAUD et GATHELIER (1970) et POUYAUD (1970) ont déjà amplement décrit les particularités de ces bassins et en particulier de celui très marécageux de la Likouala aux Herbes. Les formations de couverture de cette région sont constituées d'alluvions quaternaires fluviatiles argileuses ou sableuses.

Exception de la présence au sud-ouest des plateaux Tékés, la Cuvette centrale est entourée de la grande forêt équatoriale recouvrant de l'ouest au nord, des reliefs émoussés et peu élevés $(400-800 \mathrm{~m}$ ) où affleure le socle continental africain composé de roches cristallines et cristallophyliennes. Au sud et à l'est, cette forêt pluviale se prolonge au-delà de la dépression zaïroise pour couvrir dans son ensemble près de $48 \%$ de la totalité du bassin du fleuve.

Pour les plateaux Tékés, le pluviogramme interannuel moyen de Djambala (figure 2a), fait état d'une saison " sèche " principale bien marquée entre juillet et août, alors que la deuxième saison sèche, simple inflexion dans l'ensemble de la saison des pluies, se situe entre décembre et février. La station pluviométrique de Ouesso (figure $2 b$ ) au sein de la Cuvette, illustre le climat équatorial de transition (L'HOTE et al., 1995), marqué aussi par deux saisons des pluies et deux saisons sèches inégales, mais avec une inversion entre petite et grande saison sèche, puisque la grande saison sèche est moins intense alors que la petite saison sèche est plus marquée que sur les Plateaux Tékés.

\subsection{Données disponibles}

Les onze stations hydrométriques étudiées disposent de chroniques de quarante années de relevés limnimétriques quotidiens (1953-1993). Deux stations pluviométriques possédant plus de trente années d'observations ont été sélectionnées pour leur représentativité spatiale, celle de Djambala pour les plateaux Tékés et celle de Ouesso pour la Cuvette Congolaise.

Enfin, pour les dix stations hydrométriques principales, des prélèvements mensuels d'eau ont été effectués durant les années 1992 et 1993 au sein du Programme d'Étude de la Géosphère Intertropicale - opération Grands Bassins Fluviaux (PEGV/GBF) (OLIVRY et BOULEGUE, 1995). Une partie de ces données proviennent de LARAQUE et al. (1994). 


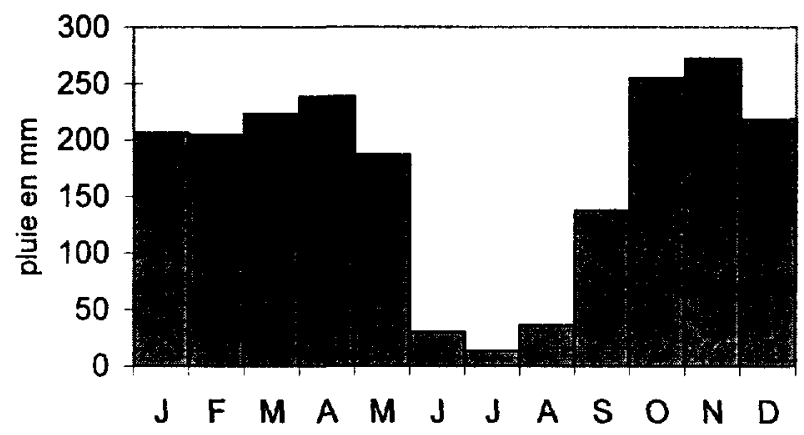

Figure 2a Régime pluviométrique des plateaux Tékés - station de Djambala (196992).

Mean rainfall charts of the Tékés Plateaus.

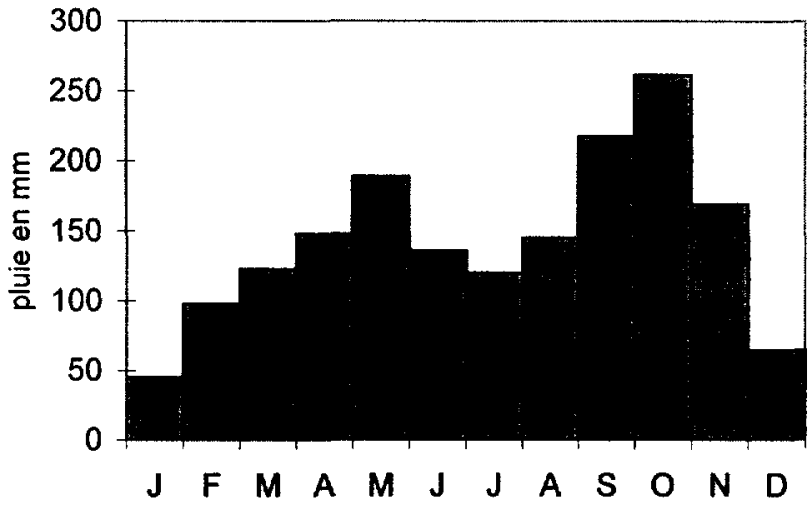

Figure 2b Régime pluviométrique de la cuvette congolaise - station de Ouesso (196694).

Mean rainfall charts of the Congolese basin.

\subsection{Protocoles}

Les échantillons mensuels d'eau étaient prélevés au milieu du cours d'eau et à $50 \mathrm{~cm}$ de la surface. Pour les cours d'eau tropicaux, l'erreur relative due à ce mode de prélèvement n'excède pas $10 \%$ de la valeur obtenue à partir d'une intégration des mesures effectuées sur toute une verticale (NOUVELOT, 1969).

Étant donné les caractéristiques physiographiques des milieux étudiés (superficies des bassins versant souvent très supérieures à $5000 \mathrm{~km}^{2}$, régime pluviométrique équatorial, présence d'aquifère important, etc.) qui entraînent une certaine lenteur des crues et décrues, cette fréquence d'échantillonnage s'est révélée suffisante pour enregistrer les variations temporelles de composition des eaux, qui sont relativement faibles dans de tels écosystèmes.

- Les mesures du $\mathrm{pH}$, de la conductivité électrique (compensées à $25^{\circ} \mathrm{C}$ ) et de la température ont été réalisées in situ à partir d'appareils portatifs (analyseur multiparamètres à affichage digital type Knick Portames digital pH metter 651-2). 
- Un tamisage à $50 \mu \mathrm{m}$ permet de séparer les matières en suspension grossières (Mesg) assimilées aux sables et une filtration à $0,2 \mu \mathrm{m}$ isole les matières en suspension fines (Mesf).

- Les dosages des éléments minéraux dissous ont été réalisés par le Laboratoire des Formations Superficielles de l'ORSTOM de Bondy, comme suit :

- $\mathrm{Ca}, \mathrm{Mg}, \mathrm{Na}, \mathrm{K}$, par absorption atomique (limite de détection de $0,01 \mathrm{mg} \cdot \mathrm{L}^{-1}$ ),

- $\mathrm{Cl}, \mathrm{NO}_{3}, \mathrm{SO}_{4}$, par chromatographie ionique (limite de détection de $\left.0,1 \mathrm{mg} \cdot \mathrm{L}^{-1}\right)$,

- $\mathrm{SiO}_{2}, \mathrm{Al}_{2} \mathrm{O}_{3}, \mathrm{Fe}_{2} \mathrm{O}_{3}$, par spectrographie d'émission de plasma (limite de détection de $0,02 \mathrm{mg} \cdot \mathrm{L}^{-1}$ ),

- Enfin, le titre alcalimétrique complet, est déterminé par dosage potentiométrique (limite de détection de $0,5 \mathrm{mg} \cdot \mathrm{L}^{-1}$ ).

\section{2 - RÉSULTATS ET DISCUSSION}

\subsection{Classification hydrologique régionale}

Lidentité régionale de chacun des deux domaines et leur différenciation, précédemment établies sur la base de critères physiographiques et climatiques, doit être examinée sur le plan de leur fonctionnement hydrologique. Pour cela, nous avons comparé deux paramètres hydrologiques qui ne présentent pas de liens physiques entre eux mais qui illustrent bien les particularités de leurs régimes hydrologiques.

II s'agit d'une part du coefficient d'écoulement interannuel moyen :

$$
\text { CE }(e n \%)=\left[\Sigma_{1}{ }^{n}\left(L E_{i} / P_{i}\right) / n\right] \times 100 \text {, }
$$

Avec $L E_{i}=$ lame d'eau écoulée pour l'année i en mm,

$P_{i}=$ précipitation moyenne de l'année $i$ en $\mathrm{mm}$,

et d'autre part du coefficient d'irrégularité saisonnière moyen interannuel des écoulements :

$$
\text { CIS }=\Sigma_{1}{ }^{n}\left(Q_{\max } / Q \min _{i}\right) / n,
$$

avec $Q_{\max }=$ débit mensuel maximum de l'année i, et $Q \min _{i}=$ débit mensuel minimum de l'année i, $n=$ nombre d'années étudiées et $i=$ indice variant entre 1 et $n$.

La figure 3 , représentant chacune des rivières des plateaux Tékés et de la Cuvette à leur principale station hydrométrique en fonction de ces deux critères, permet de dégager nettement deux nuages de points. Un premier ensemble compact de valeurs correspond à des coefficients d'écoulement compris entre $45 \%$ et $60 \%$ et des coefficients d'irrégularité saisonnière entre 1,1 et 1,5 et un deuxième groupe plus allongé pour lequel les mêmes valeurs se situent respectivement dans des intervalles de $20 \%$ à $30 \%$ et de 2,5 à 5,5 . Les coefficients d'irrégularité saisonnière pris année par année sur la période d'étude, sont bien moins dispersés pour le premier groupe avec des valeurs extrêmes de 1,04 et 1,7 , contre 1,2 et 12 pour le second (tableau 1). 
Tableau 1 Données hydrologiques et physico-chimiques des cours d'eau étudiés.

Hydrological and chemical data of the studied rivers.

\begin{tabular}{|c|c|c|c|c|c|c|c|c|c|c|c|c|c|c|c|c|}
\hline \multirow{2}{*}{\multicolumn{2}{|c|}{ Rivières }} & \multirow{2}{*}{ Stations } & \multirow{2}{*}{ SBV } & \multirow{2}{*}{ Qmst } & \multirow{2}{*}{ VARAN } & \multicolumn{3}{|c|}{ VARSI } & \multirow{2}{*}{ COEC } & \multirow{2}{*}{ as } & \multirow{2}{*}{ PER1 } & \multirow{2}{*}{ CTO } & \multirow{2}{*}{ SDT } & \multirow{2}{*}{ CE } & \multirow{2}{*}{$\mathbf{p H}$} & \multirow{2}{*}{ PER2 } \\
\hline & & & & & & moy. & $\max$ & $\min$. & & & & & & & & \\
\hline Djoué (a) & & Goma Tsé-Tsé & 5740 & 137 & 1,64 & 1,41 & 2,20 & 1,20 & 48,30 & 23,87 & $1953-93$ & 13,77 & 22,56 & 23,95 & 7,26 & $1992-93(1)$ \\
\hline Djiri (a) & & Pont RN2 & 930 & 25 & 1,23 & 1,13 & 1,45 & 1,01 & 53,00 & 26,88 & $1983-93$ & 1,08 & 8,91 & 5,21 & 5,83 & $1992-93(1)$ \\
\hline Léfini (a) & & Mbouambé & 13500 & 416 & 1,22 & 1,24 & 1,69 & 1,17 & 59,90 & 30,81 & $1953-93$ & 2,86 & 10,60 & 7,95 & 5,94 & $1992-93(1)$ \\
\hline Nkéni (a) & & Gamboma & 6200 & 200 & 1,21 & 1,12 & 1,26 & 1,09 & 59,60 & 32,26 & $1953-93$ & 2,05 & 10,11 & 6,13 & 5,66 & $1992-93(1)$ \\
\hline Alima (a) & & Tchicapika & 20070 & 585 & 1,24 & 1,28 & 1,62 & 1,04 & 52,90 & 29,15 & $1953-93$ & 2,40 & 10,44 & 8,04 & 5,68 & $1992-93(1)$ \\
\hline Kouyou (a) & & Ewo & 1030 & 35 & 1,30 & 1,33 & 1,54 & 1,22 & 56,00 & 33,98 & $1963-77$ & - & - & - & - & - \\
\hline Kouyou (b) & & Owando & 10100 & 215 & 1,99 & 2,14 & 3,93 & 1,88 & 37,90 & 21,29 & $1963-77$ & 4,06 & 11,49 & 11,16 & 5,69 & $1992.93(2)$ \\
\hline Likouala Mossal & aka (c) & Makoua & 14100 & 215 & 2,27 & 3,34 & 8,81 & 2,42 & 29,10 & 15,25 & $1953-93$ & 12,17 & 23,65 & 20,15 & 6,47 & $1992-93(2)$ \\
\hline Mambili (c) & & Yengo & 12080 & 190 & 2,47 & 2,54 & 4,60 & 1,20 & 29,90 & 15,73 & $1953-93$ & 27,85 & 38,27 & 38,40 & 6,90 & $1992-93(2)$ \\
\hline Sangha (c) & & Ouesso & 158300 & 1616 & 1,93 & 3,79 & 8,09 & 1,96 & 20,80 & 10,21 & $1953-93$ & 39,40 & 51,48 & 54,30 & 6,94 & $1992-93(2)$ \\
\hline Likouala aux $\mathrm{He}$ & erbes (c) & Botouali & 24800 & 285 & 2,32 & 5,36 & 12,00 & 3,65 & 21,20 & 11,49 & $1953-93$ & 11,08 & 17,84 & 23,94 & 5,36 & $1992-93(2)$ \\
\hline $\begin{aligned} & \text { Legende: } \text { (a) } \\
& \text { (b) } \\
& \text { (c) } \\
& \text { SBV } \\
& \text { OmSt } \\
& \text { VARAN } \\
& \text { VARSI } \\
& \text { COEC } \\
& \text { QS }\end{aligned}$ & $\begin{array}{l}=\text { rivière } \\
=\text { rivière } \\
=\text { rivière } \\
=\text { superf } \\
=\text { débits } \\
=\text { variabi } \\
=\text { variabi } \\
=\text { coetfic } \\
=\text { débits }\end{array}$ & $\begin{array}{l}\text { s des plateaux Téké } \\
\text { s mixtes } \\
\text { ficie bassin versant } \\
\text { moyens à la station } \\
\text { ilité interannuelle d } \\
\text { ilité saisonnière des } \\
\text { cient d'écoulement } \\
\text { spécifiques en L.s }\end{array}$ & $\begin{array}{l}\text { golaise } \\
\text { en } \mathrm{km}^{2} \\
n \mathrm{en} \mathrm{m}^{3} . \mathrm{s}^{-1} \\
\text { es modules } \\
\text { s modules } \\
\text { annuel moye } \\
-1 . \mathrm{km}^{2}\end{array}$ & nen $\%$ & & $\begin{array}{l}\text { PER1 } \\
\text { CTD } \\
\text { SDT } \\
\text { CE } \\
\text { PER2 } \\
\text { PER2 (1) } \\
\text { PER2 (2) } \\
\text { PH }\end{array}$ & $\begin{array}{l}=\text { péri } \\
=\text { char } \\
=\text { soli } \\
=\text { CTD } \\
=\text { con } \\
=\text { péri } \\
\text { ) sou } \\
\text { l) sou } \\
=\text { d } 25\end{array}$ & $\begin{array}{l}\text { ge totale } \\
\text { les disso } \\
+\mathrm{SiO}_{2}+ \\
\text { luctivité } \\
\text { de de su } \\
\mathrm{ce}: \text { Lara } \\
\mathrm{ce}: \text { résu } \\
{ }^{\circ} \mathrm{C}\end{array}$ & $\begin{array}{l}\text { lissoute er } \\
\text { s totaux e } \\
\mathrm{A}_{2} \mathrm{O}_{3}+\mathrm{Fe} \\
\text { ectrique e } \\
\text { vi physico } \\
\text { ue et al., ( } \\
\text { ats du pro }\end{array}$ & $\begin{array}{l}\text { jique quot } \\
\mathrm{mg} . \mathrm{L}^{-1} \\
\mathrm{mg} \cdot \mathrm{L}^{-1} \\
\mathrm{O}_{3} \\
\mathrm{\mu S} \cdot \mathrm{cm}^{-1} \\
\text { chimique } \\
994) \\
\text { jramme P }\end{array}$ & $\begin{array}{l}\text { idien } \\
\text { à } 25^{\circ} \mathrm{C} \\
\text { mensuel } \\
\text { EGI/GBF }\end{array}$ & & & & & \\
\hline
\end{tabular}




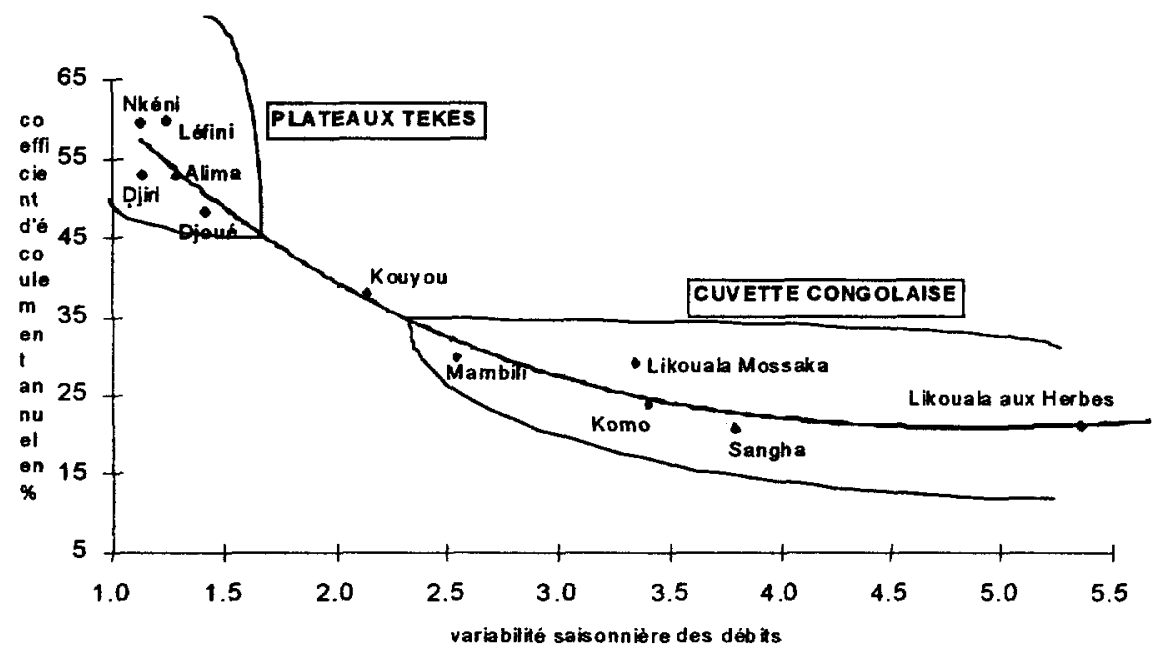

Figure 3 Classification hydrologique des affluents congolais du fleuve Congo.

Hydrological classification of the Congolese tributaries of the Congo River.

Cette opposition est une fois encore celle des rivières Tékés (Djiri, Léfini, Nkéni, Alima, Djoué) et des cours d'eau de la Cuvette congolaise. On sait que les premières avec en particulier la Nkéni, comptent parmi les rivières les plus régulières de la planète. Ces rivières ont des modules de 25 à $600 \mathrm{~m}^{3} \cdot \mathrm{s}^{-1}$ pour des bassins versant de 1000 à $20000 \mathrm{~km}^{2}$ (LARAQUE et MAZIEZOULA, 1995). Leurs hydrogrammes (figure 4a) sont extraordinairement réguliers : ni hautes eaux, ni étiages malgré la présence d'une saison pluviométrique sèche bien marquée de juin à août (figure 2a). La remarquable régularité de leur régime hydrologique a d'ailleurs été notée très tôt par bon nombre d'auteurs (BABET, 1947, LE MARÉCHAL, 1966, OLIVRY, 1967, SCHWARTZ, 1988). Il en est de même pour la Lekony à Akieni au Gabon (CARRÉ, 1978), affluent de rive droite du cours supérieur de l'Ogoué qui dessert la portion occidentale des grès Tékés avec une régularité remarquable (variabilité saisonnière de 1,11 pour un coefficient d'écoulement

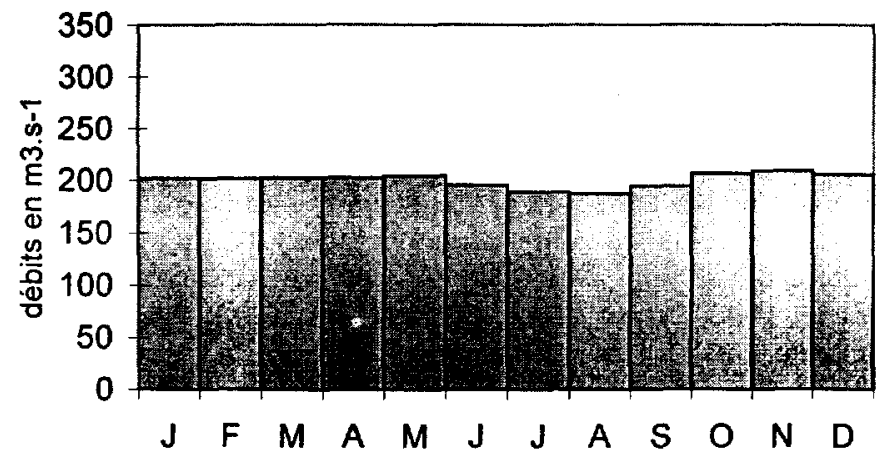

Figure 4a Hydrogramme moyen de la Nkéni à Gamboma (période 1953-93).

Hydrological regimes of the Nkéni River at Gamboma. 


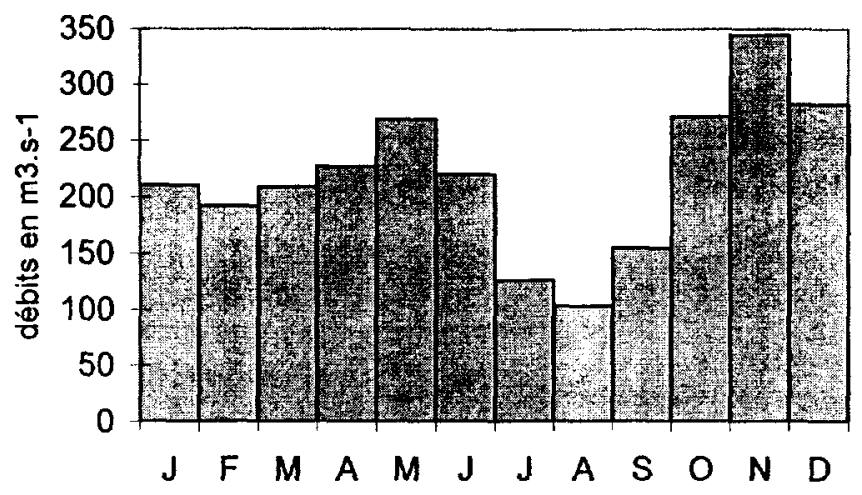

Figure 4b Hydrogramme moyen de la Likouala Mossaka à Makoua (période 1953-93).

Hydrological regimes of the Likouala Mossaka River at Makoua.

interannuel de $60 \%$ ). Ces faibles variations saisonnières moyennes des débits mensuels ne peuvent s'expliquer que par une grande capacité de stockage et donc de régulation de l'aquifère sablo-gréseux, dont on a souligné précédemment qu'il est bien alimenté par infiltration des eaux pluviales. Cette régulation se traduit également par une remarquable constance des débits moyens annuels (ou modules), comme l'attestent ceux de la Nkéni représentés en figure $5 \mathrm{a}$. En effet, les hydraulicités (rapport des modules sur le module interannuel), de ces rivières avoisinent en général 1,2 (tableau 1). Cet aquifère rend compte aussi d'une certaine inertie interannuelle des réponses hydrologiques face à la baisse récente des précipitations de ces dernières années, baisse il est vrai, plus modérée que celle enregistrée dans la bande tropicale humide de transition située plus au Nord (MAHÉ, 1993, LARAQUE et OLIVRY, 1995). Enfin, les valeurs des coefficients d'écoulement des rivières Tékés sont élevées (45 à $60 \%$ ) ainsi que celles de leurs débits spécifiques ( 25 à $35 \mathrm{~L} . \mathrm{s}^{-1} \cdot \mathrm{km}^{-2}$ ).

Les cours d'eau du domaine forestier (Likouala aux Herbes, Sangha, Komo, Likouala Mossaka, Mambili), dont les débits aux principales stations hydrométriques vont de 25 a $1600 \mathrm{~m}^{3}$. $\mathrm{s}^{-1}$ pour des superficies variant de 1000 à 160000 $\mathrm{km}^{2}$ (LARAQUE et MAZIEZOULA, 1995), ont des régimes hydrologiques bien différents. Les coefficients d'écoulement (20 à $30 \%)$ et les débits spécifiques (10 à $15 \mathrm{~L} . \mathrm{s}^{-1} . \mathrm{km}^{-2}$ ) sont plus de deux fois inférieurs à ceux des Plateaux Tékés, alors que les totaux pluviométriques ne baissent que de $10 \%$ entre ces deux régions pour passer de $1800-2000 \mathrm{~mm}$.an ${ }^{-1}$ sur les Plateaux à $1600-1800 \mathrm{~mm}$.an-1 sur la Cuvette (BULTOT, 1971). Par contre, les variations interannuelles d'hydraulicité illustrées sur la figure $5 \mathrm{~b}$ par l'exemple de la Likouala aux Herbes, sont plus importantes et comprises entre 1,9 et 2,5 suivant les cours d'eau. Ces derniers ont en outre été plus influencés depuis une vingtaine d'années par la baisse pluviométrique qui a davantage affecté les régions soudano-sahéliennes (SIRCOULON, 1987, MAHÉ, 1993, OLIVRY et al., 1993, OLIVRY, 1993). Pour l'ensemble de la cuvette congolaise, LARAQUE et OLIVRY (1995) ont calculé à partir de la comparaison des modules des périodes 1987-93 et 1953-93, une baisse d'hydraulicité de $11 \%$. Leurs débits présentent aussi des variabilités saisonnières plus élevées (coefficients d'irrégularité de 2,5 à 5,5 ) que sur les Plateaux Tékés. Cette dernière dispersion est en fait plus courante en hydrologie et dépend des caractéristiques physiographiques des bassins versants. 


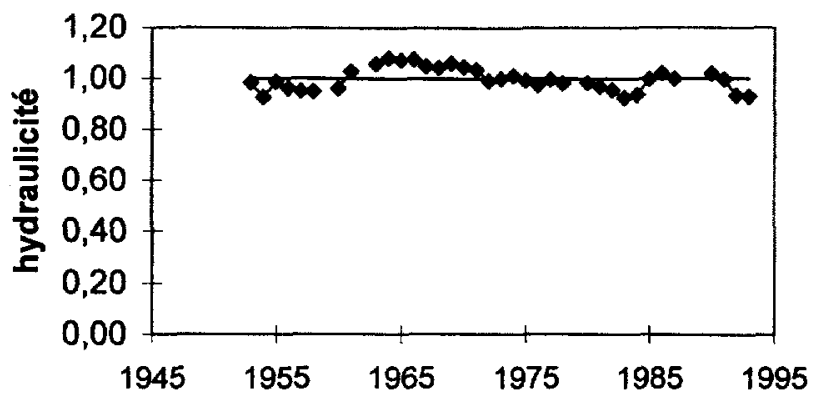

Figure 5a Évolution de l'hydraulicité de la Nkéni à Gamboma.

Evolution of the "annual discharge/interannual discharge ratio" of the Nkeni River at Gamboma.

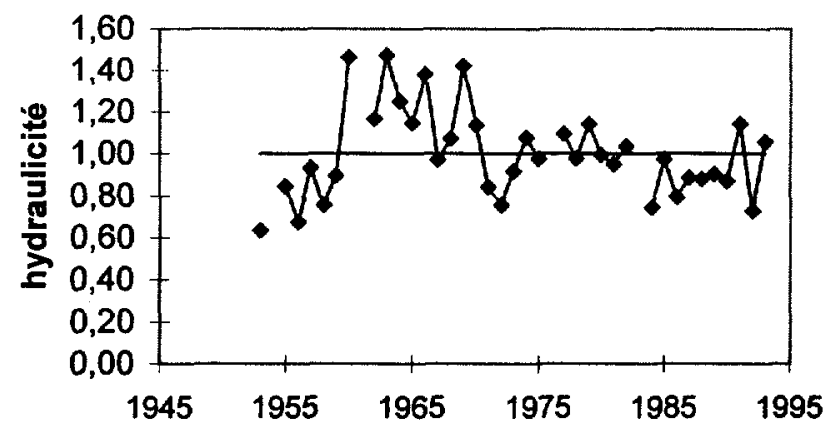

Figure 5b Évolution de l'hydraulicité de la Likouala aux Herbes à Botouali.

Evolution of the "annual discharge/interannual discharge ratio" of the Likouala aux Herbes River at Botouali.

La moindre perméabilité des terrains, l'interception des eaux pluviales et l'évapotranspiration par le couvert forestier, ainsi que l'évaporation directe sur les zones inondables et les marécages, entraînent un déficit d'écoulement important. En l'absence d'un aquifère, du moins aussi imposant et régulateur que celui des plateaux Tékés, leurs régimes hydrologiques reflètent plus fidèlement le rythme pluviométrique régional (figures $2 b$ et $4 b$ ). Ce n'est pas le cas des régimes hydrologiques Tékés, insensibles à la pluviométrie contrastée des Plateaux, qui fait notamment état d'une saison sèche bien marquée de trois mois.

Malgré la physiographie plus hétérogène de son bassin amont très étendu (160 $000 \mathrm{~km}^{2}$ ), la Sangha à Ouesso présente des comportements similaires induits en grande partie par le sous-bassin forestier du Dja-Ngoko, affluent provenant du Cameroun et dont le cours inférieur marque la frontière avec le Congo. Ce dernier présente encore des caractéristiques hydrologiques similaires à celles observées dans la Cuvette avec des débits spécifiques à sa confluence de $11,3 \mathrm{~L} . \mathrm{s}^{-1} \cdot \mathrm{km}^{-2}$ pour une variabilité saisonnière des débits un peu plus élevée, de l'ordre de 6,4 .

Dans l'ensemble de ces affluents de rive droite du fleuve Congo, le Kouyou mérite une attention particulière. II se singularise nettement par un coefficient 
d'irrégularité saisonnière de 2,1 , un coefficient d'écoulement égal à $40 \%$ et un débit spécifique de $21 \mathrm{~L} . \mathrm{s}^{-1} \cdot \mathrm{km}^{-2}$. Ces valeurs le placent en position intermédiaire par rapport aux deux groupes principaux, ce qui s'explique fort logiquement par l'influence partagée spatialement, de l'aquifère gréseux sur le flanc sud-ouest de son bassin et la présence de la grande forêt équatoriate au nord-est. Ce cours d'eau marque en quelque sorte la limite entre ces deux milieux naturels, aux dynamiques de fonctionnement différentes. Le changement de son régime hydrologique entre les parties amont et aval de son bassin en est lillustration. En effet, son cours supérieur, suivi à la station hydrométrique d'Ewo (tableau 1), présente le régime régulier caractéristique des rivières des plateaux auquel se substitue à l'aval, à la station d'Owando, le régime de la Cuvette aux fluctuations saisonnières plus importantes (figure 6).

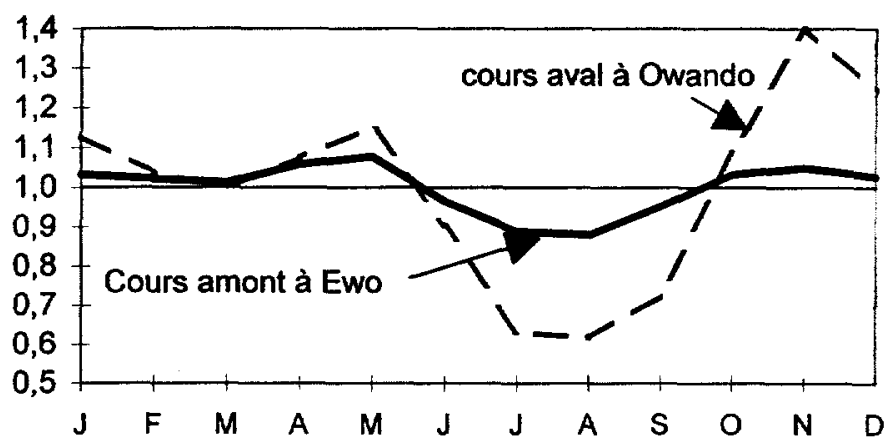

Figure 6 Variations mensuelles des régimes des cours supérieur et inférieur du Kouyou.

Seasonal variations of the up-and down-stream regimes of the Kouyou River.

\subsection{Typologie hydrochimique}

À partir des principaux résultats d'analyse d'eau, l'on constate que les deux groupes d'affluents précédemment individualisés s'opposent aussi par leur biogéochimie. Les " rivières blanches » des plateaux Tékés sont très peu minéralisées avec des conductivités électriques de l'ordre de 5 à $8 \mu \mathrm{S} . \mathrm{cm}^{-1}$ à $25^{\circ} \mathrm{C}$ (tableau 1), les ions solubles des formations tertiaires ayant été lessivés en quasi totalité. C'est en raison de l'exceptionnelle pauvreté en ions minéraux (1 à $\left.3 \mathrm{mg} \cdot \mathrm{L}^{-1}\right)$, que la proportion de silice est si élevée ( $75 \%$ de la minéralisation totale) alors que ses teneurs (8-10 mg. $\mathrm{L}^{-1}$ ) restent voisines de la moyenne mondiale de 10,4 mg.L-1 (BERNER et BERNER, 1987).

En effet, les données de LARAQUE et al., (1994), montrent que les cations $\mathrm{Ca}^{++}, \mathrm{Mg}^{++}, \mathrm{K}^{+}$et les anions $\mathrm{HCO}_{3}^{-}, \mathrm{Cl}^{-}, \mathrm{SO}_{4}=\mathrm{NO}_{3}^{-}$, classés par ordre de concentration décroissante, ne participent en fait que pour $25 \%$ de la minéralisation totale de ces eaux (figure 7). Certains éléments ont même des teneurs proches de celles des eaux de pluies. C'est le cas par exemple de la Léfini qui présente des teneurs en chlorures de 5 à 5,5 mmoles.L-1 (NEGREL, 1992).

En ce qui concerne les eaux des rivières de la Cuvette congolaise dites " noires ou brunes ", la phase minérale est très différente tant par la charge 


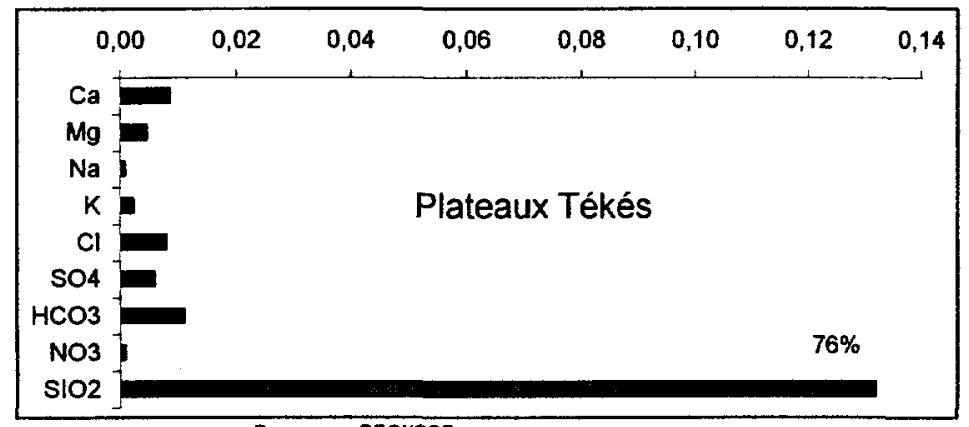

source : Programme PEGI/GBF

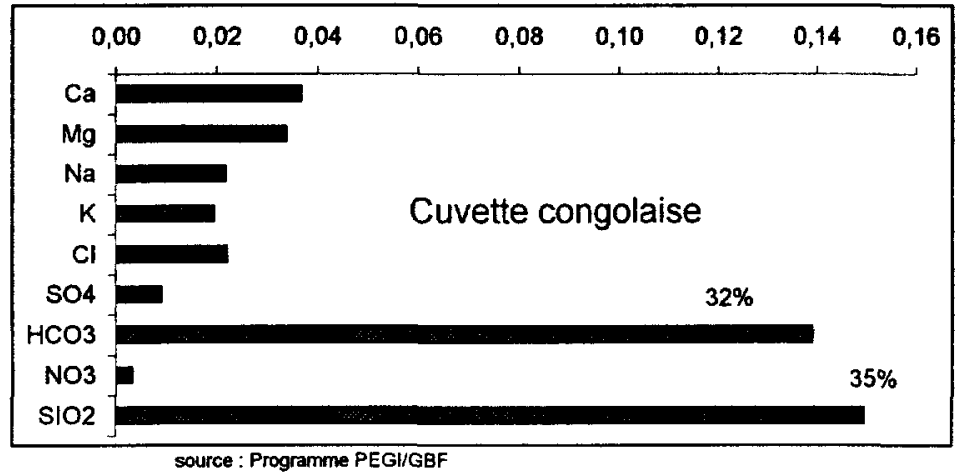

Figure 7 Composition des phases minérales dissoutes (concentrations moyennes en mmoles. $L^{-1}$ ) - période 1992-93.

Composition of dissolved mineral phases (mean concentration in mmol...t) - period 1992-93.

globale qu'au niveau des concentrations respectives des divers éléments. Si la concentration moyenne en silice est quasi identique à celle des rivières Tékés (environ 0,14 mmoles. $L^{-1}$ ), cette partie de la phase dissoute ne représente plus que $35 \%$ de la minéralisation totale. Les teneurs en éléments alcalino-terreux et plus particulièrement en alcalins, prennent des valeurs plus grandes, alors que $\mathrm{HCO}_{3}-$ voit sa concentration multipliée par 15 pour atteindre 0,15 mmoles. $\mathrm{L}^{-1}$, soit $32 \%$ de la phase minérale dissoute. Le taux de chlorures double tandis que celui des anions sulfates reste identique. Cette différenciation hydrochimique entre les cours d'eau des deux régions trouve très probablement son explication dans la nature lithologique des formations géologiques respectives et aussi dans les différences de couverts végétaux. Les bassins forestiers présentent en effet des substrats plus variés soumis à une pédogenèse plus active et les charges minérales moyennes de leurs rivières se situent généralement entre 17 et $40 \mathrm{mg} \cdot \mathrm{L}^{-1}$. Pareil résultat se retrouve aussi sur le flanc sud des plateaux Tékés, sur le bassin du Djoué, affluent du Congo, dont les eaux contiennent $22 \mathrm{mg} . \mathrm{L}^{-1}$ de matières minérales dissoutes. En effet, son bassin présente des formations géologiques plus différenciées (LARAQUE et al., 1994) et sa couverture végétale est également partagée entre forêt et savane.

Les couvertures végétales, savane d'une part et forêt de l'autre, détermineraient elles aussi de notables différences dans la biochimie des eaux. Sur les pla- 
teaux aux savanes herbacées, les teneurs en carbone organique sont de $4,7 \mathrm{mg} . \mathrm{L}^{-1}$ (SEYLER et al., 1995) contre $25,5 \mathrm{mg} \cdot \mathrm{L}^{-1}$ dans la cuvette. Les acides fulviques et humiques allochtones sont à l'origine de l'acidité des " eaux noires " de la Cuvette centrale avec parfois des $\mathrm{pH}$ inférieurs à 4 unités.

Quant à la Sangha, de par l'extension de son bassin versant et son hétérogénéité physiographique, elle se détache quelque peu des grandeurs communément rencontrées dans la Cuvette inondable. Sa conductivité électrique moyenne est de $55 \mu \mathrm{S} . \mathrm{cm}^{-1}$ à $25^{\circ} \mathrm{C}$ pour des pH oscillant autour de la neutralité entre 6,8 et 7,2 , et ses eaux sont par conséquent bien plus riches en bicarbonates (près de $50 \%$ ).

Si les taux d'exportation de matières dissoutes spécifiques sont faibles, ils sont en revanche semblables entre les deux domaines géographiques malgré les écarts importants de concentration. Pour les matières d'origines minérale et orga-

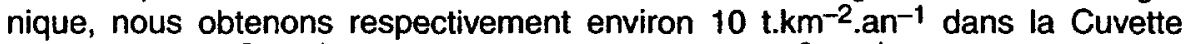
contre $9,7 \mathrm{t} \mathrm{km}^{-2}$.an-1 dans les Plateaux et 19,3 t. $\mathrm{km}^{-2}$.an-1 en forêt contre 13,5 t. $\mathrm{km}^{-2}$. an ${ }^{-1}$ pour la savane. Cette similitude apparente est évidemment liée à la compensation par les débits spécifiques plus élevés des rivières des Plateaux, de l'ordre, rappelons-le, de $30 \mathrm{~L} . \mathrm{s}^{-1} . \mathrm{km}^{-2}$, soit plus de deux fois supérieurs à ceux de la Cuvette.

\section{CONCLUSION}

Parmi les affluents congolais de rive droite, deux familles se distinguent. Aux rivières claires très peu minéralisées des Plateaux Tékés s'opposent les « eaux noires " de la Cuvette congolaise aux eaux acides et relativement riches en matière organique. Les premières se caractérisent par des coefficients d'écoulement et des débits spécifiques particulièrement élevés avec des hydrogrammes très réguliers où les variations saisonnières sont insignifiantes malgré un régime pluviométrique contrasté. C'est le contraire pour les " eaux noires " aux régimes pluviométrique et hydrométrique semblables. Formations géologiques et couvertures végétales de ces deux entités géographiques seraient à l'origine des différences hydrologiques. Les rivières Tékés sont issues d'un important aquifère régulateur sablo-gréseux couvert de savanes, qui infiltre très bien les eaux météoritiques à son sommet pour les restituer ensuite à sa base, tandis que les cours d'eau de la cuvette congolaise circulent sous une forêt pluviale dense et sur des alluvions à la lithologie plus variée, sans bénéficier d'une telle régulation hydrogéologique. 


\section{RÉFÉRENCES BIBLIOGRAPHIQUES}

BABET V., 1947. Compte rendu de la réunion des géologues du Congo occidental, tenue à Léopoldville les 10, 11 et 12 décembre 1945 - Bull. Serv. des Mines, Paris, 3, 72 p.

BERNER, BERNER, 1987. The global water cycle. Geochemistry and environment. Prentice Hall, 397 p.

BULTOT F., 1971. Atlas climatique du bassin congolais - les composantes du bilan de l'eau - t.2 - I.N.E.A.C., Bruxelles.

BURGIS M.J., SYMOENS J.J., 1987. African wetlands and shallow water bodies Zones humides et lacs peu profonds d'Afrique, ORSTOM, Paris, 1987,650 p.

CARRÉ P., 1978. Fleuves et rivières du Gabon - Diversité des régimes hydrologiques. CENAREST, Libreville, Gabon, $24 \mathrm{p}$.

COSSON, 1955. Notice explicative sur les feuilles de Pointe Noire et Brazzaville. Carte de reconnaissance au 1/500 000, Gouv. géné. AEF; Direction des Mines, Brazzaville.

LARAQUE A., BRICQUET J.P., OLIVRY J.C., BEATHELOT M., 1993. Export of matter from the Congo river (PEGl program) - in proceeding of the first International Symposium on Perspectives for Environmental Geochemistry in Tropical Countries, Niteroï, Brazil, nov. 29-dec. 3, 1993: 327-332.

LARAQUE A., PANDI A., BERTHELOT M., MIETTON M., 1994. Écoulement et transport de matière dans le bassin versant du Djoué : affluent du fleuve Congo-Zaïre Hydrologie continentale, 9, $2: 10 \mathrm{p}$.

LARAQUE A., MAZIÉZOULA B., 1995. Banque de données hydrologiques des affluents congolais du fleuve Congo-Zaïre et informations physiographiques, rapport interne, lab. Hydr.ORSTOM, Montpellier, $250 \mathrm{p}$.

LARAQUE A., OLIVRY J.C., 1995. Évolution de l'hydrologie du Congo-Zaîre et de ses affluents rive droite et dynamique de ses transports solides et dissous - dans Géologie tropicale: géosciences et outil pour le développement. Actes de la Conférence de Paris, mai 1995. Rev. AlHS, $238: 271-288$.
LE MARÉCHAL A., 1966. Contribution à l'étude des plateaux Batékés (géologie, géomorphologie, hydrogéologie), OASTOM, Brazzaville, $42 \mathrm{p}$.

L'HOTE Y., DUBREUIL P., LERIQUE J., 1995. Carte des types de climat en Afrique Noire à l'ouest du Congo - Rappels et extension aux régimes hydrologiques dans Géologie tropicale: géosciences et outil pour le développement. Acles de la Conférence de Paris, mai 1995. Rev. AlHS, 238 : 55-65.

MAHÉ G., 1993. Les écoulements fluviaux sur la façade atlantique de l'Afrique - étude des éléments du bilan hydrique et variabilité interannuelle, analyse de situations hydroclimatiques moyennes et extrêmes Thèse de doctorat - Coll. études et thèses ORSTOM, Paris, $438 \mathrm{p}$.

NEGREL P., 1992. Utilisation du Strontium, des alcalins et alcalino-terreux pour la détermination des bilans des éléments chimiques dans les fleuves: apports atmosphériques, altération des roches exemple du bassin du Congo - Thèse de doctorat - Univ Paris 7, 249 p.

NOUVELOT J.F., 1969. Mesure et étude des transports solides en suspension au Cameroun. Cah. Orstom, série Hydrologie, 6 (4) : 43-86.

OLIVRY J.C., 1967. Régime hydrologique des rivières Batéké. Rap. Orstom, Brazzaville, nov. 1967, 27 p.

OLIVRY J.C., BOULEGUE J., 1995. Grands bassins fluviaux périatlantiques: Congo, Niger, Amazone, (Actes du colloque PEGI -INSU-CNRS-ORSTOM, du 22 au 24 novembre 1993), éd. Orstom, Paris, France, 505 p.

OLIVRY J.C., BRICQUET J.P., MAHÉ G., 1993. Vers un appauvrissement durable des ressources en eau de l'Afrique humide? - Hydrology of warm humids regions - proceeding of the Yokohama Symposium, IAHS, july 1993, 6 p.

OLIVRY J.C., 1993. Évolution récente des régimes hydrologiques des grands fleuves d'Afrique de l'Ouest et Centrale - in Les écosystèmes intertropicaux, fonctionnement et usages - journées du programme Environnement CNAS/ORSTOM, 13, 14 et 15 janvier 1993, Lyon, $9 \mathrm{p}$. 
POUYAUD B., GATHELIER R. (1970). Les rivières du bassin de la Likouala Mossaka. Centre ORSTOM, Brazzaville, $48 \mathrm{p}$.

POUYAUD B., 1970. La Likouala aux Herbes à Epéna et à Botouali. Centre ORSTOM, Brazzaville, $37 \mathrm{p}$.

SCHWARTZ D., 1988. Histoire d'un paysage : Le Lousséké. Paléoenvironnements quaternaires et podzolisation sur sables Batéké (Quarante derniers millénaires, région de Brazzaville, R.P. du Congo). These doctorat ès Sciences, Uni. Nancy !, in Coll. Etudes et Thèses, ORSTOM, Paris, $285 \mathrm{p}$.

SEYLER P., ETCHEBER H., ORANGE D., LARAQUE A., SIGHA-NKAMDJOU L.,
OLIVRY J.C., 1993. Contributions, fluctuations saisonnières et flux de carbone dans le bassin du Congo. Actes du colloque PEGl - Grands bassins fluviaux périatlantiques : Congo, Niger, Amazone (INSU-CNRS-ORSTOM), Paris, France, du 22 au 24 novembre, p. 217-228.

SIRCOULON J., 1987. Variations des débits des cours d'eau et des niveaux des lacs en Afrique de l'Ouest depuis le $20^{\mathrm{e}}$ siècle. In The Influence of climate change and climatic variability on the hydrologic regime and water ressources (Proceedings of the Vancouver Symposium. August, 1987), IAHS Publication $n^{\circ} 168$, p 13-25. 